\title{
Limited expression of APRIL and its receptors prior to intestinal IgA plasma cell development during human infancy
}

\author{
CE Gustafson ${ }^{1,2,3}$, D Higbee ${ }^{1,3}$, AR Yeckes $^{4}$, CC Wilson $^{1,2}$, EF De Zoeten $^{4}$, P Jedlicka ${ }^{5}$ and EN Janoff ${ }^{1,2,3}$
}

The absence of immunoglobulin A $(\lg A)$ in the intestinal tract renders young infants highly susceptible to enteric infections. However, mediators of initial IgA induction in this population are undefined. We determined the temporal acquisition of plasma cells by isotype and expression of T cell-independent (TI) and -dependent (TD) IgA class switch factors in the human intestinal tract during early infancy. We found that IgA plasma cells were largely absent in the infant intestine until after 1 month of age, approaching adult densities later in infancy than both IgM and IgG. The restricted development of IgA plasma cells in the first month was accompanied by reduced expression of the TI factor a proliferation-inducing ligand (APRIL) and its receptors TACI (transmembrane activator and calcium-modulator and cyclophilin ligand interactor) and B cell maturation antigen (BCMA) within isolated lymphoid follicles (ILFs). Moreover, both APRIL and BCMA expression strongly correlated with increasing IgA plasma cell densities over time. Conversely, TD mediators (CD40 ligand (CD40L) and CD40) were expressed within ILFs before 1 month and were not associated with IgA plasma cell generation. In addition, preterm infants had lower densities of IgA plasma cells and reduced APRIL expression compared with full-term infants. Thus, blunted TI responses may contribute to the delayed induction of intestinal IgA during early human infancy.

\section{INTRODUCTION}

Immunoglobulin A ( $\operatorname{Ig} \mathrm{A})$ predominates in the intestinal tract and is crucial for modulation of commensal microbiota and defense against enteric pathogens. ${ }^{1,2}$ The absence of mucosal IgA renders newborns particularly susceptible to intestinal infections. Initially, newborns and infants may acquire protection through passive transfer of maternal IgG via the placenta and secretory IgA in maternal breast milk, but they must generate their own mucosal IgA to maintain intestinal defense after weaning.

IgA-secreting cells in the infant intestinal tract are absent at birth but increase with age. ${ }^{3-5}$ The development of these IgAproducing cells requires recruitment of $B$ cells to organized lymphoid structures within the mucosa, class switch recombination (CSR) from IgM to IgA and differentiation to antibodysecreting plasma cells. In mice and humans, intestinal IgA CSR in naïve $\mathrm{B}$ cells occurs classically through $\mathrm{T}$ cell-dependent
(TD) mechanisms within gut-associated lymphoid tissue such as Peyer's patches and isolated lymphoid follicles (ILFs). ${ }^{6-8}$ In these inductive sites, CSR to IgA follows engagement of CD40 on $\mathrm{B}$ cells with $\mathrm{CD} 40 \mathrm{~L}$ from $\mathrm{CD} 4^{+}$helper $\mathrm{T}$ cells and upregulation of the CSR-inducing enzyme activation-induced cytidine deaminase (AID) in B cells. ${ }^{6}$ Additionally, helper T cells can provide non-cognate help by secreting immunomodulating cytokines such as transforming growth factor $\beta$ (TGF $\beta$ ), interleukin 10 (IL-10), and IL-6 that augment B cell CSR, differentiation, and survival, respectively., ${ }^{6,9}$

Alternatively, T cell-independent (TI) mechanisms of IgA CSR in the intestinal tract of mice, and possibly of humans, are mediated by interactions of $\mathrm{B}$ cells with CD40L-like molecules, a proliferation-inducing ligand (APRIL) and B cell-activating factor (BAFF). ${ }^{10,11}$ These molecules are produced locally by cells within the intestinal tract, including epithelial cells, dendritic cells, macrophages, and neutrophils, ${ }^{12}$ and directly

\footnotetext{
Mucosal and Vaccine Research Program Colorado (MAVRC), Infectious Disease Division, University of Colorado Anschutz Medical Campus, Aurora, Colorado, USA ${ }^{2}$ Integrated Department of Immunology, National Jewish Health, University of Colorado Anschutz Medical Campus, Aurora, Colorado, USA. ${ }^{3}$ Department of Medicine, Denver Veterans Affairs Medical Center, Denver, Colorado, USA. ${ }^{4}$ Department of Pediatrics, University of Colorado Anschutz Medical Campus, Aurora, Colorado, USA and ${ }^{5}$ Department of Pathology, School of Medicine, University of Colorado Anschutz Medical Campus, Aurora, Colorado, USA. Correspondence: EN Janoff (edward.janoff@ucdenver.edu)
} 
engage surface receptors on $\mathrm{B}$ cells (B cell maturation antigen (BCMA), TACI (transmembrane activator and calciummodulator and cyclophilin ligand interactor), and BAFF-R) to induce the upregulation of AID and IgA class switching. ${ }^{13,14}$ Indeed, mice deficient in APRIL and humans lacking a receptor for APRIL (TACI), both exhibit substantially reduced IgA production. ${ }^{15,16}$ However, mice lacking $\mathrm{T}$ cells, CD4, or CD40L still develop relatively normal intestinal IgA responses, ${ }^{10,11,17}$ highlighting the importance of TI, rather than TD, mechanisms of CSR in intestinal IgA development.

The sequence of and specific requirements for mucosal IgA development have been elegantly characterized in mouse models, but mice and humans show anatomical and cellular differences in their IgA induction systems. ${ }^{18}$ Mice have a single IgA isotype and require TGF $\beta$ signaling for IgA class switching. ${ }^{19}$ Unlike mice, humans have two subclasses of $\operatorname{IgA}$, IgA1 and IgA2, and do not require TGF $\beta$ stimulation for IgA class switching. ${ }^{13}$ Additionally, human newborns have ILFs, a site of IgA CSR, in the intestinal tract at birth whereas newborn mice only develop ILFs in response to bacterial exposure after birth. Given the multiple developmental differences in IgA CSR between mice and humans, the requirements for the induction of IgA plasma cells in the human newborn may differ from those observed in newborn mouse models, among which TI CSR appears to be predominate.

Although the human adult intestinal tract expresses all the components necessary for both TD and TI class switching, ${ }^{12}$ the contributions of TD and/or TI CSR in the generation of intestinal IgA plasma cells in human infants are not well characterized. Defining the ontogeny of IgA plasma cells from birth and the contributions of TD and TI IgA class switch factors within the human infant intestinal tract will facilitate the identification of potential targets for enhancing early infant IgA responses to enteric pathogens and mucosal vaccines. Thus, we characterized the localization and densities of intestinal plasma cells and their isotype distribution in the first 2 years of life. We further determined the expression of factors in the intestine required for CSR (AID) and mucosal TD $\left(\mathrm{CD} 4^{+} \mathrm{T}\right.$ cells, CD40L, and CD40) and TI (APRIL, BAFF, TACI, BMCA, and BAFF-R) class switching in the human infant. We show that TI class switch factors APRIL and BCMA, but not TD factors, have limited expression within ILFs during the first month of life but increase over time, in association with the progressive accumulation of IgA plasma cells in the human infant intestinal tract.

\section{RESULTS}

\section{Cytoplasmic $\lg \mathrm{A}^{+}\left(\operatorname{clg} \mathrm{A}^{+}\right)$plasma cells reach adult densities after those with $\mathrm{clgM}^{+}$or $\mathrm{clgG}^{+}$in the infant intestinal tract}

In the adult intestinal tract, B cells are localized to inductive sites, such as ILFs and non-inductive isolated lymphoid aggregates (ILAs), whereas plasma cells are found in the effector site, the lamina propria (LP) ${ }^{20}$ Consistent with this study, we found that infant $\mathrm{CD} 20^{+} \mathrm{B}$ cells were only detected within or in very close proximity to ILFs and ILAs, but not within the LP or among epithelial cells. Conversely, plasma cells, when present, were predominant in the LP (Supplementary Figure 1 online). The density of infant $\mathrm{CD} 138^{+}$plasma cells in the LP increased progressively over the first 2 years of life (Figure 1a), with no plasma cells detectable until after 1 week of age. Plasma cells were present in all infants greater than 2 months of age (range: $85-1,566$ cells per $\mathrm{mm}^{2}$ ). Although plasma cell densities increased significantly by $1-2$ months and remained higher than $0-1$ month densities throughout the 2 years, they did not approach values observed in adults until after 6 months. Hence, although localization of plasma cells mirrored that of adults, the acquisition of intestinal plasma cells in infants is largely delayed until after 1 month of age.

Adult intestinal plasma cells primarily express IgA. Because we observed increasing plasma cell densities over time, we next investigated plasma cell isotypes (cytoplasmic $\operatorname{IgA}\left(\operatorname{cIgA}^{+}\right)$, $\mathrm{cIgM}^{+}$, and $\mathrm{cIgG}^{+}$) in the infant intestinal tract from birth to 2 years by immunofluorescence. Similarly to $\mathrm{CD} 138^{+}$plasma cell densities, $\mathrm{cIg}^{+}$cells increased over time in the infant LP, accounting for $28 \%$ (range: $17-41 \%$ ) of total LP cells by 2 years of age (Figure 1b, data not shown). However, the distribution of individual isotypes varied over time. $\operatorname{cIgA}{ }^{+}$cell densities most closely mirrored those of $\mathrm{CD} 138^{+}$cells (Figure 1c), both of which steadily increased over time.

In infant sera, IgM is produced earlier in life than IgA. ${ }^{21}$ Consistent with these results in the infant systemic compartment, the densities of intestinal $\mathrm{cIgM}^{+}$cells attained adult levels earlier than $\operatorname{did} \operatorname{cIgA}{ }^{+}$cells and even exceeded adult densities between 6 and 24 months ( $218.5 \pm 42.8$ vs. $120.3 \pm$ 26.4 cells per $\mathrm{mm}^{2}$, mean \pm s.e.m.) (Figures $1 \mathrm{c}$ and d). Additionally, the mean density of $\mathrm{cIgM}^{+}$cells was higher between 0 and 1 month, albeit marginally, than that of $\operatorname{cIgA}^{+}$ cells ( $45.8 \pm 24.7$ and $38.5 \pm 15.6$ cells per $\mathrm{mm}^{2}$, respectively). Compared with adults, $\mathrm{cIgM}^{+}$cells represented a larger proportion of intestinal plasma cells throughout the first 2 years of life (Figure 1f). cIgG $^{+}$cells were present at very low frequencies both throughout the first 2 years and in adults (Figure 1e). Nevertheless, by 2 months, both the densities and proportions of cIgA ${ }^{+}$cells exceeded those of both $\operatorname{cIgM}^{+}$and $\mathrm{cIgG}^{+}$cells and remained the predominant isotype thereafter (Figure 1f).

To confirm that the cIgA ${ }^{+}$cells observed in the infant tissues were indeed plasma cells $\left(\mathrm{Ki}^{-} 7^{-}\right)$and not recently migrated plasmablasts $\left(\mathrm{Ki} 7^{+}\right)$, we stained for the proliferation marker Ki67 by immunofluorescence. No cIgA ${ }^{+}$cells in the infant LP had detectable nuclear $\mathrm{Ki} 7^{+}$staining (Figure 1g), confirming that $\mathrm{cIgA}^{+}$cells are indeed terminally differentiated plasma cells. Of note, $\mathrm{Ki}^{+} 7^{+}$staining was only detectable within ILFs and the epithelium in the infant intestinal tract.

\section{AID mRNA, localized to ILFs, is expressed before IgA plasma cell development}

Because $\mathrm{B}$ cells must first undergo CSR to become $\operatorname{IgA}{ }^{+}$plasma cells, we next determined the localization of AID mRNA, an essential enzyme in B cell CSR, in the infant intestinal tract. Previous research in the human intestine suggests that AID 

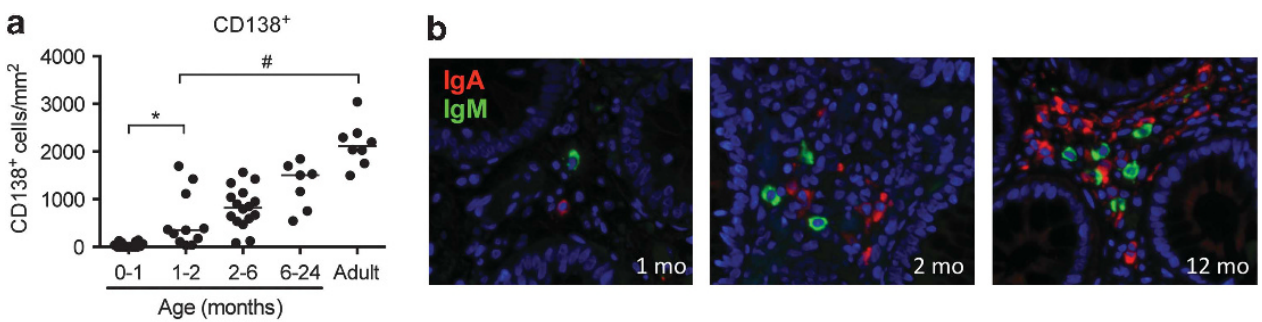

C
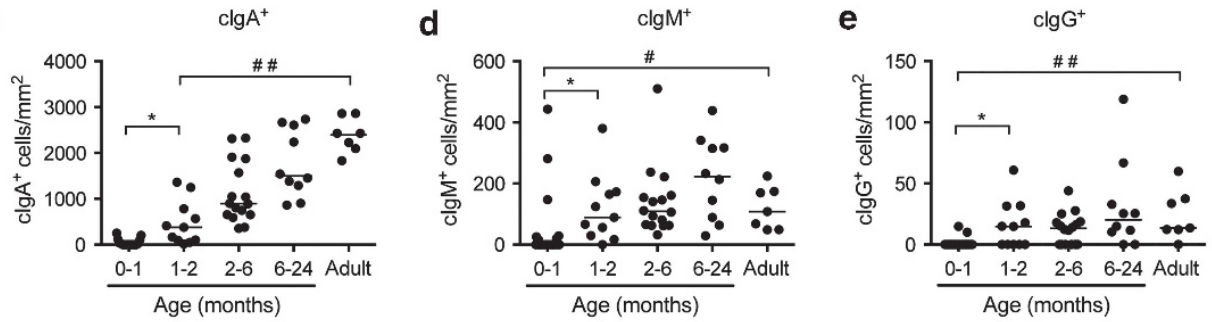

f Isotype distribution
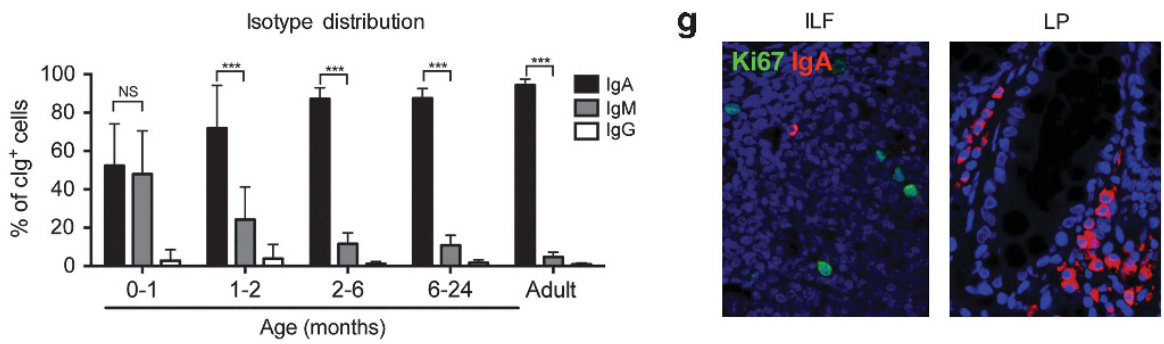

Figure 1 Development of plasma cells in the infant intestinal tract. (a) CD138 ${ }^{+}$cells per $\mathrm{mm}^{2}$ in the lamina propria of infant biopsies. Samples were separated into age groups ( $n=16,11,16,7$, and 8 , respectively, from 0 to 1 month to adult). Each dot represents the average number of CD138 ${ }^{+}$cells per $\mathrm{mm}^{2}$ in an individual sample. (b) Representative cytoplasmic IgM (clgM) (green) and IgA (red) immunofluorescent staining of infant biopsies at 1, 2, and

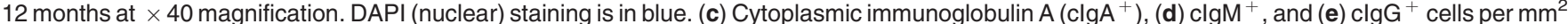
in lamina propria of infant biopsies $\left(n=21,11,16,10\right.$, and 7 , respectively, from 0 to 1 month to adult). Horizontal black lines indicate medians. ${ }^{\star} P<0.05$ compared with 0-1 month proportions. ${ }^{\#} P<0.05$, ${ }^{\# \#} P<0.001$, compared with adults. (f) Percent of $\operatorname{clgA}^{+}$, clgM ${ }^{+}$, and clgG ${ }^{+}$cells compared with total $\mathrm{Ig}^{+}$cells. ${ }^{* \star *} P<0.0001$, comparing the $\operatorname{lgA}$ and $\operatorname{lgM}$ percentages in each age group. Bars represent mean + s.d., all $P$-values were determined using Kruskal-Wallis followed by Dunn's multiple comparison. NS = not significant. (g) Representative Ki67 (green) and IgA (red) staining in infant intestinal tract within isolated lymphoid follicles (ILF) and the lamina proria (LP). DAPI (nuclear) staining is in blue.
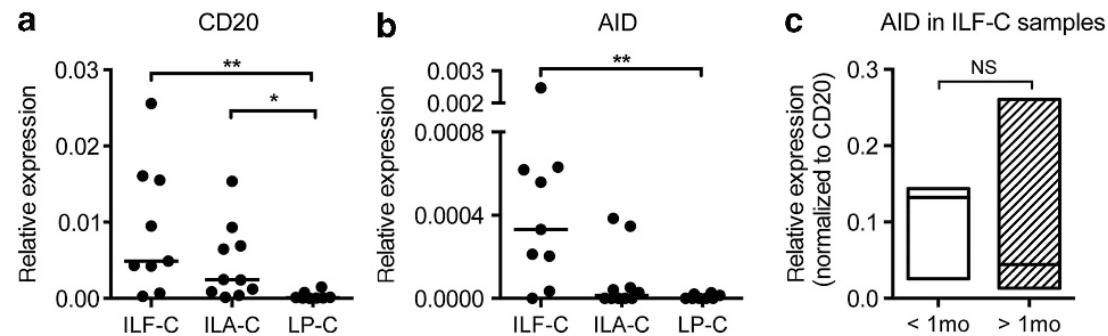

Figure 2 Localization and temporal expression of activation-induced cytidine deaminase (AID) in the infant intestinal tract. (a) CD20 and (b) AID mRNA expression was determined by quantitative reverse transcription PCR (qPCR) in infant biopsies (0-6 months of age). Tissue samples are divided into three groups: (1) those containing isolated lymphoid follicles (ILFs) (ILF-C, $n=9$ ), (2) those containing ILAs but not ILFs (ILA-C, $n=10)$, or (3) those containing neither ILAs nor ILFs (lamina propria (LP)-C, $n=8$ ). ${ }^{*} P<0.05,{ }^{\star *} P<0.01$. $P$-values were determined using Kruskal-Wallis followed by Dunn's multiple comparison, medians shown. (c) AID expression normalized to CD20 in ILF-C samples less than $(<1$ mo; $n=3)$ or greater than 1 month $(>1 \mathrm{mo} ; n=6)$. Bars indicate median with $\min$ and max values. $\mathrm{NS}=$ not significant.

expression is localized exclusively to organized lymphoid structures (i.e., ILFs) and is not detectable within nonorganized lymphoid structures (i.e., ILAs) or the LP. ${ }^{12,22,23}$ Thus, we compared AID and CD20 mRNA expression in infants ranging from 0 to 6 months of age with quantitative reverse transcription PCR ( $\mathrm{qPCR}$ ). We divided the infant samples into three histologic groups: (i) those containing ILFs
(ILF-containing, termed as ILF-C), (ii) those containing B cell ILAs but not ILFs (ILA-containing, termed as ILA-C), and (iii) those containing LP but not ILFs or ILAs (LP-containing, termed as LP-C). CD20 mRNA expression was higher in ILF-C and ILA-C samples than in LP-C samples, which showed very little if any CD20 expression (Figure 2a), consistent with the localization of CD20 staining (Supplementary Figure 1). 

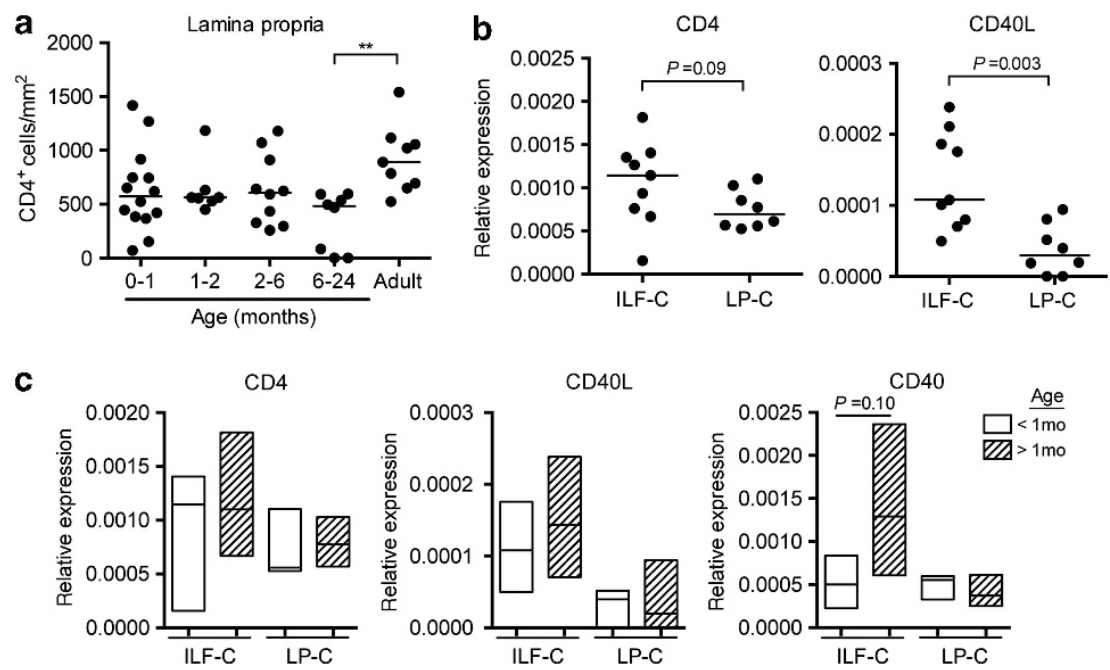

Figure 3 Localization and temporal expression of T cell-dependent factors in the infant intestinal tract. (a) Lamina propria CD4 ${ }^{+} \mathrm{T}_{\text {-cell }}$ densities were determined by immunohistochemistry $(n=14,7,10,8$, and 9 , respectively, from 0 to 1 month to adult). Black lines indicate medians. $P$-values were determined using Kruskal-Wallis with Dunn's post-test. ${ }^{\star \star} P<0.01$. (b) CD4 and CD40 ligand (CD40L) expression was determined by quantitative reverse transcription PCR (qPCR) in infant intestinal tissues, ages ranging 0-6 months, in isolated lymphoid follicle (ILF)-C ( $n=9)$ and lamina propria (LP)-C $(n=8)$ samples. (c) CD4, CD40L, and CD40 mRNA expression in samples less than $(<1 \mathrm{mo} ; n=3)$ or greater than 1 months $(>1 \mathrm{mo} ; n=5-6)$. Bars indicate median with min and max values. $P$-values were determined using Mann-Whitney $U$-test.

Similarly, ILF-C samples showed significantly higher AID mRNA expression than LP-C samples (Figure 2b). Indeed, expression of AID was virtually absent in all LP-C samples and present in only 2 of 10 ILA-C samples.

Consistent with our observation that the development of $\mathrm{cIgA}^{+}$cells was delayed compared with $\operatorname{cIgM}^{+}$cells in the LP of young infants, we hypothesized that AID expression in infant $B$ cells would be reduced in the first months of life. However, we found that AID expression, when normalized to either GAPDH or CD20, was similar between samples obtained at less than or greater than 1 month of age (Figure 2c, data not shown). Surprisingly, AID was expressed in ILF-C samples before 1 month, even though very few IgA plasma cells were present in this time period. Therefore, AID expression, which is present before 1 month of age, does not appear to be restricting the generation of infant intestinal IgA plasma cells.

\section{TD factors are present in the infant intestinal tract before IgA plasma cell development}

Although AID is required for CSR, its expression is not sufficient to support the generation of IgA plasma cells. Additional factors are utilized by both TD and TI mechanisms to promote CSR to IgA and downstream IgA plasma cell development. To identify the factors involved in these pathways that could contribute to IgA CSR in young infants, we determined the localization and the temporal development of TD factors $\left(\mathrm{CD} 4^{+} \mathrm{T}\right.$ cells, $\mathrm{CD} 40 \mathrm{~L}$, and $\left.\mathrm{CD} 40\right)$ in the infant intestinal tract. $\mathrm{CD} 4{ }^{+} \mathrm{T}$ cells were present in the earliest samples within the LP and ILFs, as determined by immunohistochemistry (IHC) (Figure 3a, data not shown). However, unlike IgA plasma cells, $\mathrm{CD} 4^{+} \mathrm{T}$ cells in the LP did not increase in density with infant age. Similarly, CD4 mRNA expression was detectable at comparable levels in LP-C and ILF-C samples at birth and did not change with age (Figures $3 \mathbf{b}$ and $\mathbf{c}$ ). CD40L and CD40 were expressed in LP-C and ILF-C samples and both were higher in ILF-C samples (Figures $\mathbf{3 b}$ and $\mathbf{c}$ ). Temporally, CD40L and CD40 were expressed before 1 month and did not change over time in ILF-C or LP-C samples, although CD40 expression did trend toward higher expression after 1 month in ILF-C tissues.

We additionally determined whether specific cytokines important for IgA CSR (TGF $\beta$ ), B cell differentiation (IL-10) and plasma cells survival (IL-6) were expressed in the infant intestinal tract. TGF $\beta$, IL-10, and IL- 6 were all expressed in LP-C and ILF-C samples and showed no change in expression level with age (data not shown). Thus, although IgA development is delayed until after 1 month of age, B cell and $\mathrm{T}$ cell factors essential for CD40-CD40L mediated CSR, B cell differentiation, and plasma cell survival are already present in the infant intestinal tract before IgA plasma cells.

TI factor APRIL and its receptors TACI and BCMA have significantly reduced expression in the first month of life Given the early expression of TD components, we hypothesized that factors involved in TI CSR may instead be absent or reduced in the infant intestinal tract during the first months of life. Thus, we determined the localization and expression of APRIL, BAFF, and their receptors (TACI, BCMA, and BAFFR) by qPCR in samples between 0 and 6 months. APRIL and BAFF were expressed both in LP-C and in ILF-C samples at all ages. However, levels of APRIL, but not BAFF, increased significantly after 1 month within ILF-C samples (Figure 4a). Of note, APRIL and BAFF were expressed at consistent levels within LP-C samples over time, indicating their alternative role in plasma cell survival within the intestinal LP is likely preserved during early infancy. 

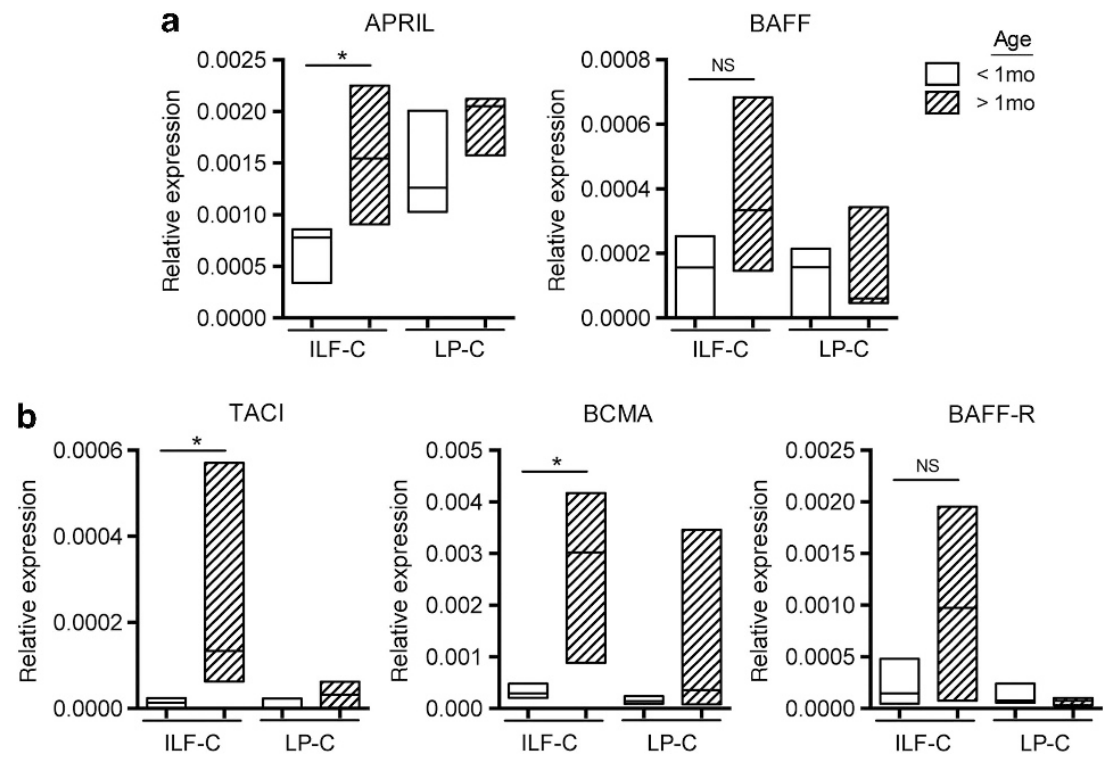

Figure 4 T cell-independent factor localization and temporal expression in the infant intestinal tract. (a) A proliferation-inducing ligand (APRIL), B cellactivating factor (BAFF), and (b) their receptors transmembrane activator and calcium-modulator and cyclophilin ligand interactor (TACl), B cell maturation antigen (BCMA), and BAFF-R mRNA expression in isolated lymphoid follicle (ILF)-C and lamina propria (LP)-C samples was determined using quantitative reverse transcription PCR (qPCR). Samples were divided into age groups; less than 1 month of age $(<1 \mathrm{mo} ; n=3)$ or greater than 1 months $(>1 \mathrm{mo} ; n=5-6)$. Bars indicate median with min and max values. $P$-values were determined using Mann-Whitney $U$-test. ${ }^{*} P<0.05$. NS $=$ not significant.

Unlike APRIL and BAFF expression, TACI was virtually absent in all LP-C samples but present in ILF-C samples (Figure $4 \mathrm{~b}$ ), consistent with TACI being expressed by $\mathrm{B}$ cells and not by plasma cells. On the other hand, BCMA, which can be found on both B cells and plasma cells, ${ }^{24}$ was expressed in both LP-C and ILF-C samples. Similarly to APRIL, both TACI and BCMA expression increased significantly after 1 month in ILF-C samples. BAFF-R, an additional receptor for BAFF, showed no significant changes over time, although its expression did appear reduced before 1 month of age. Thus, decreased expression of APRIL and its receptors BCMA and TACI within ILFs may impair IgA plasma cell development in the infant intestinal tract during the first month of life.

\section{Expression of APRIL and its receptor BCMA within ILFs correlates with increasing IgA plasma cell densities in the infant intestinal tract}

To identify potential mediators of IgA plasma cell development, we determined the relationships between plasma cell densities and ILF-associated expression of TD and TI CSR factors in the infant intestinal tract utilizing a multiple comparison approach (see Methods). Our data divided into two clusters: cluster one included IgA, CD138, age, APRIL, and BCMA; and cluster two included CD20, CD40, TACI, and BAFF-R (Supplementary Figure 2). Indeed, APRIL and BCMA expression within ILF-C samples strongly correlated with IgA plasma cell densities (Figure 5a), whereas TACI and BAFF-R expression correlated with B cell-specific CD20 expression (Figure 5b). Although TACI expression did not directly correlate with $\operatorname{cIgA}^{+}$cell densities, samples lacking TACI expression exhibited no detectable $\operatorname{cIgA}^{+}$cells (data not shown). Thus, the interaction between APRIL and its receptors TACI and BCMA on B cells may have a critical role in IgA plasma cell induction during early infancy.

Since APRIL expression within ILFs most strongly correlated with infant IgA plasma cell development, we determined the presence and localization of APRIL $^{+}$cells in comparison with $B$ cell localization within ILFs in infant intestinal tract by IHC. Soluble APRIL and APRIL ${ }^{+}$cells were preferentially detectable within the B cell area of ILFs in the infant intestinal tract by IHC (Figures $\mathbf{5 c}$ and $\mathbf{d}$ ), highlighting that APRIL-producing cells are present and in contact with B cells in infant ILFs. Additionally, cells in the ILF epithelial dome stained more intensely for APRIL than adjacent epithelial cells (Figure 5e). Consistent with our mRNA expression data, $\mathrm{APRIL}^{+}$cells were also identified within the LP (Figure 5f). Therefore, the interaction between APRIL-producing cells and B cells expressing APRILspecific receptors (TACI and BCMA) within ILFs in the infant intestinal tract may be facilitating the induction of intestinal IgA during early infancy.

Preterm infants have fewer intestinal plasma cells than fullterm infants, with reductions in both IgA plasma cells and APRIL expression

Breastfeeding and gestational age (GA) can influence infant immune development and thus could be affecting the induction of intestinal IgA plasma cells. We were unable to evaluate the effect of breastfeeding on IgA development in this cohort as the majority of these infants were either exclusively breastfed or mixed fed at the time of biopsy. However, we had sufficient numbers of preterm $(n=6$, less than 37 weeks GA) and full-term infants ( $n=8$, greater than 37 weeks GA) samples ranging between 1 and 6 months of age (median age: 2.11) to compare IgA plasma cell development. Age range, gender, and 
a

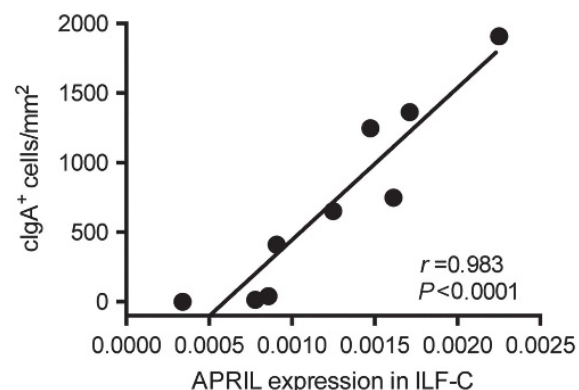

b

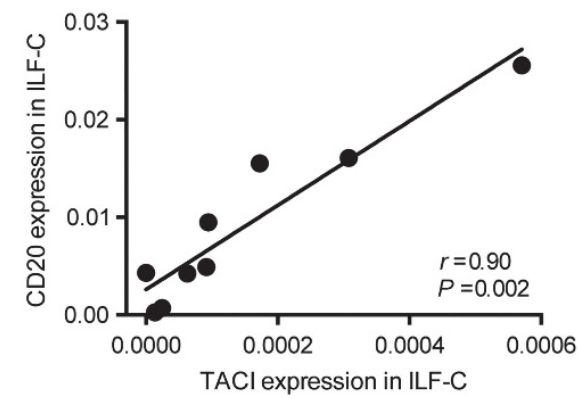

C

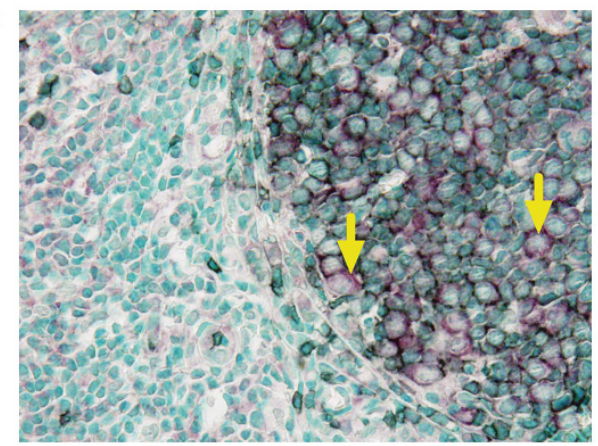

e

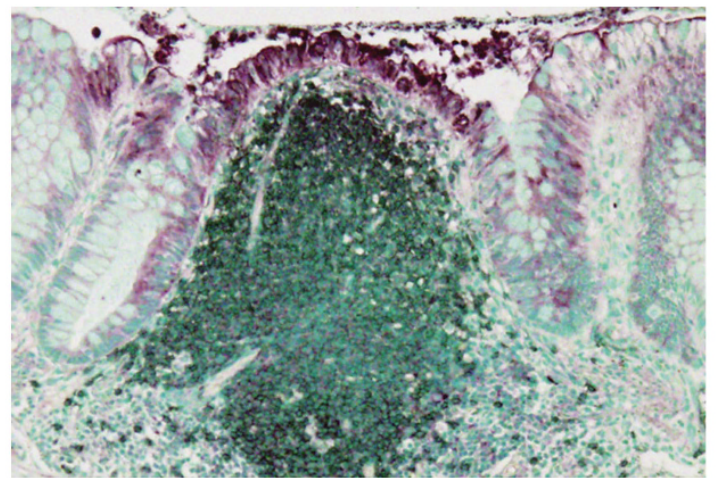

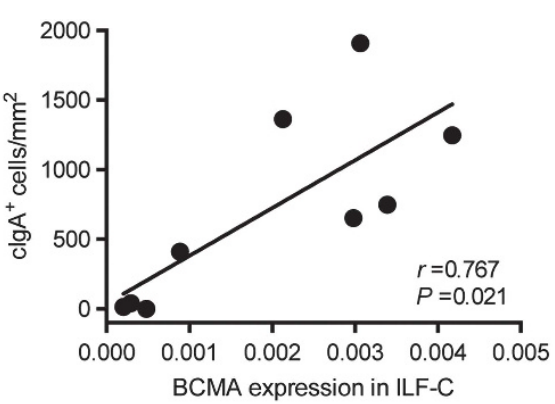

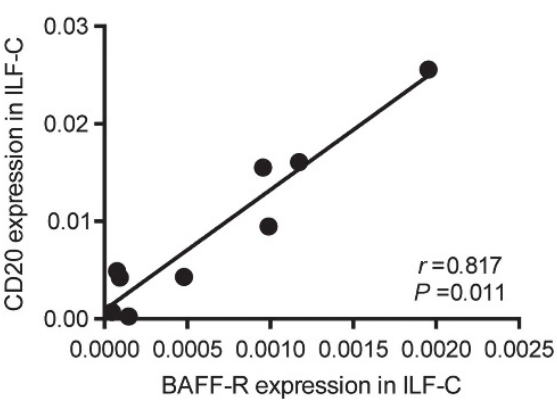

d
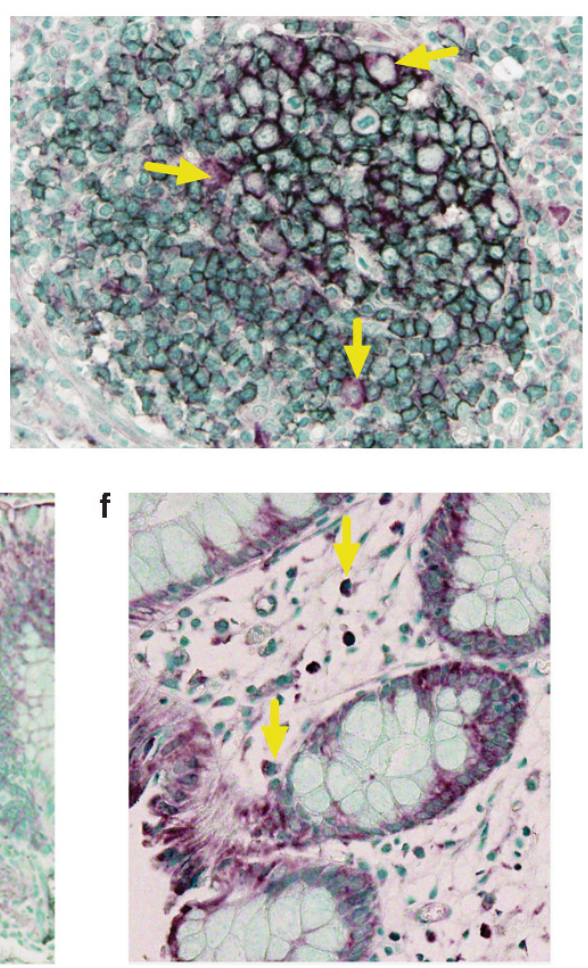

Figure 5 Relationship between cytoplasmic immunoglobulin $\mathrm{A}\left(\mathrm{clg} \mathrm{A}^{+}\right)$cells, B cells and $\mathrm{T}$ cell-independent factors in the infant intestinal tract. Correlations of (a) a proliferation-inducing ligand (APRIL) and B cell maturation antigen (BMCA) expression compared with clgA ${ }^{+}$cell densities and (b) transmembrane activator and calcium-modulator and cyclophilin ligand interactor (TACl) and B cell-activating factor (BAFF)-R compared with CD20 expression in isolated lymphoid follicle (ILF)-C samples $(n=9)$ using Spearman's rank correlation. Immunohistochemistry of APRIL (purple) and CD20 (black) within infant intestinal (c-e) isolated lymphoid follicles, $(\mathbf{e}, \mathbf{f})$ epithelium, and (f) lamina propria. Yellow arrows indicate APRIL ${ }^{+}$cells.

breastfeeding methods were similar between the two groups, although preterm infants did have a higher incidence of caesarian section delivery (Supplementary Table 1 online).

Preterm infants exhibited lower $\mathrm{CD} 138^{+}$plasma cell densities compared with full-term infants (Figure 6a). $\mathrm{cIgA}^{+}$cell densities showed a similar trend (568 vs. 1,147 median cells per $\mathrm{mm}^{2}$ ). Conversely, the densities of $\mathrm{cIgM}^{+}$cells were comparable in both groups, as were $\mathrm{CD} 4{ }^{+} \mathrm{T}$ cell densities in the LP (data not shown).

BCMA expression and subsequent IgA production after in vitro APRIL stimulation are reduced in peripheral B cells from preterm infants. ${ }^{25}$ Thus, we additionally determined APRIL and BCMA expression in a subset of preterm $(n=3)$ and full-term $(n=3)$ infants. Although there was no difference 

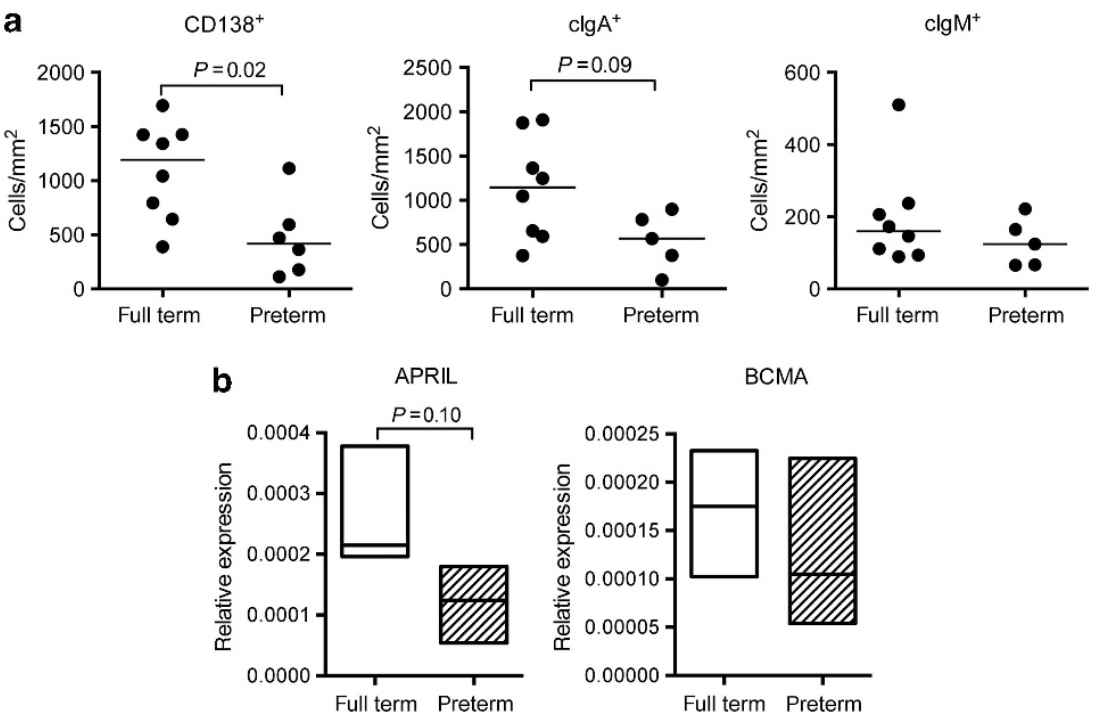

Figure 6 Effects of preterm birth on plasma cell development in the infant intestinal tract. (a) $\mathrm{CD}_{138^{+}}$, cytoplasmic immunoglobulin $\mathrm{A}$ (clgA ${ }^{+}$), and $\mathrm{clgM}^{+}$cell densities were compared using Mann-Whitney $U$-test between preterm $(n=5-6)$ and full-term $(n=8)$ infants with ages ranging from 1 to 6 months of age. (b) A proliferation-inducing ligand (APRIL) and B cell maturation antigen (BCMA) mRNA expression levels in preterm ( $n=3$ ) and fullterm infants $(n=3)$. Infants with a gestational age of less than 36 weeks were considered as preterm. Infants with a gestational age greater than 37 weeks were considered as full term.

in BCMA expression, APRIL expression was reduced in preterm samples compared with full-term samples (Figure 6b), further implicating APRIL as a key mediator of early IgA plasma cell induction in the infant intestinal tract.

\section{DISCUSSION}

Delayed development of mucosal IgA production may predispose young infants to infectious diseases, particularly when they are premature or in the absence of breastfeeding. We show limited generation of intestinal IgA plasma cell during the first month of life in human infants, approaching but not attaining adult levels by 2 years. In contrast, the lower densities of IgG and IgM cells parallel adult levels by 2 months of age and even surpass adult levels by 2 years, suggesting that IgA class switching is hindered during early infancy.

AID has a critical role in IgA class switching as well as in antibody somatic hypermutation. However, AID expression, almost exclusively detected within ILFs in the infant intestinal tract, was found before the development of IgA plasma cells. These data are consistent with a previous study that showed similar AID expression in newborns and adult peripheral B cells, ${ }^{26}$ and indicate that the ability of infant $\mathrm{B}$ cells to undergo AID-mediated CSR is not restricting intestinal IgA development. Conversely, studies on infant somatic hypermutation demonstrate very limited mutations in circulating $\operatorname{IgA}^{+} \mathrm{B}$ cells from young infants compared with adults. ${ }^{27}$ Whether AIDmediated somatic hypermutation is observed in the IgA plasma cell population during early infancy and whether mutations in IgA advance more rapidly in the infant intestine than the periphery remain currently under investigation.

Within organized inductive tissues, CSR is additionally mediated by TD and/or TI mechanisms. Variables that influence TD CSR, including the presence of $\mathrm{CD} 4{ }^{+} \mathrm{T}$ cells and expression of the T-cell activation marker CD40L and its receptor $\mathrm{CD} 40$, were detectable from the earliest times of life and did not increase over time, nor were they associated with the accumulation of $\mathrm{cIgA}{ }^{+}$plasma cells. $\mathrm{CD}^{+}{ }^{+} \mathrm{T}$ cells can also influence CSR via non-cognate interaction by the secretion of soluble factors, such as TGF $\beta$, IL-6, and IL-10. Expression of all of these cytokines was detectable in the infant intestinal tract before 1 month. Thus, neither cognate nor non-cognate CD4 ${ }^{+}$ $\mathrm{T}$ cells interaction appears to be limiting in the generation of IgA plasma cells during early infancy.

TI responses in the intestinal tract of mice are essential for the development of IgA plasma cells and are mediated in part by interactions between TACI and APRIL. Indeed, newborn mice have reduced TACI expression on B cells compared with adults, leading to poor responsiveness to APRIL stimulation. ${ }^{28}$ Similarly, in the human infant intestinal tract, the expression of TACI and APRIL within ILFs was low or absent during the first month of life. Although infants lacking TACI expression also showed very few, if any, IgA plasma cells within the LP, the expression of APRIL and its other receptor BCMA, but not TACI, strongly correlated with increasing densities of intestinal IgA plasma cells. These data suggest that TACI and BCMA expression on intestinal B cells as well as increasing expression of their ligand APRIL within ILFs are important for the initial induction of IgA class switching and the accumulation of infant intestinal plasma cells.

APRIL has a central role in IgA CSR, B cell differentiation, and long-term plasma cell survival. ${ }^{29,30}$ Thus, the limited expression of APRIL observed before 1 month could be influencing multiple steps in the development of IgA plasma cells. However, APRIL and IL-6, another important plasma cell survival factor, are expressed consistently in the LP throughout infancy. APRIL ${ }^{+}$cells within the LP are also detectable before 
1 month of age. Therefore, decreased plasma cell survival within the LP is unlikely the mechanism for limited IgA plasma cell generation during the first month of life. Although we cannot fully rule out reduced $B$ cell differentiation as a cause of limited generation of IgA plasma cells, no accumulation of class switched IgA ${ }^{+}$B cells within ILFs was observed during early infancy (data not shown) and all infant IgA plasma cells within the LP appeared to be terminally differentiated, consistent with adult studies. ${ }^{22,30}$ Taken together, these data indicate that an essential function of APRIL specifically within ILFs in the young infant intestinal tract is most likely to promote IgA CSR.

It has been well established in humans and mice that APRIL's interaction with TACI can induce IgA CSR. However, the importance of BCMA in this process is still unclear. BCMA, expressed at the highest levels on plasmablasts, is detectable on a variety of $B$ cell subsets, including mature naïve and transitional B cells in cord blood. ${ }^{24}$ Moreover, BCMA has been demonstrated to have a significant, although not essential, function in IgA production in response to APRIL stimulation in mice. ${ }^{31}$ We found BCMA expression within infant ILFs but not in the LP correlated with increasing IgA plasma cell densities, implicating a potential role for BCMA in the induction of IgA CSR as well as plasma cell survival early in life. Due to the limited tissue available in our ILF-containing samples, we were unable to perform IHC staining for BCMA within ILFs. Determining the specific cell types that express BCMA and APRIL within infant ILFs and whether these cells are reduced during early infancy warrants further examination. Additionally, investigating the functional role of BCMA in APRILinduced IgA CSR in humans will provide better insight into the mechanisms of intestinal IgA induction during early infancy.

In human infants, mucosal IgA development has been linked with microbial colonization of the intestinal tract. ${ }^{32,33}$ Similarly, IgA plasma cells arise in germ-free and newborn mice in vivo upon intestinal colonization with commensal bacteria. ${ }^{10,34}$ The mechanisms for microbial-induced IgA are thought to occur primarily through TI mechanisms. In this regard, stimulation of mouse B cells in vitro with a bacterial DNA surrogate, $\mathrm{CpG}$, induces IgA production in response to APRIL by increasing $\mathrm{B}$ cell expression of TACI. ${ }^{28}$ Moreover, CpG stimulation of human B cells causes an upregulation of both TACI and BCMA expression, ${ }^{24,35}$ implying BCMA could influence IgA CSR within the infant intestinal tract upon microbial colonization.

In infants, normal intestinal colonization can be affected by a variety of clinical factors including premature birth. ${ }^{36,37}$ Preterm infants not only have altered intestinal bacterial colonization but also, immunologically, exhibit lower expression of BCMA on circulating B cells compared with full-term infants. ${ }^{25}$ Thus, prematurity may cause impaired generation of intestinal IgA plasma cells in infants. Indeed, we found that preterm infants had lower frequencies of intestinal plasma cells than full-term infants, with reduced densities of IgA but not IgM plasma cells. Preterm infants additionally had reduced expression of APRIL within the intestinal tract. Contrary to results from infant peripheral B cells, we found no difference in intestinal BCMA expression between preterm and full-term infants, likely resulting from the constant exposure of intestinal, but not peripheral, B cells to microbial products. Thus, clinical factors, including premature birth and its related clinical consequences, may modulate intestinal IgA plasma cell development during early human infancy, potentially by modifying TI CSR mechanisms, and warrant further investigation in larger prospective cohorts.

In summary, in our comprehensive examinations of intestinal IgA plasma cell development during human infancy, we found that TI factors involved in IgA class switching, APRIL, TACI, and BCMA, but not TD factors, were reduced within ILFs in the infant intestinal tract during the first months of life. Moreover, both APRIL and BCMA expression increased over time in association with the accumulation of IgA plasma cells. Similarly, preterm infants exhibited decreased intestinal IgA plasma cell densities and APRIL expression compared with fullterm infants. Thus, restricted IgA plasma cell generation in early human infancy may be due to blunted TI responses in intestinal B cells as well as reduced numbers of APRILproducing cells, such as follicular dendritic cells, within inductive sites in the intestinal tract. Identifying mechanisms by which TI mediators can be upregulated in the intestine of young infants could provide potential targets for enhancing $\operatorname{Ig} \mathrm{A}$ responses to mucosal vaccines during early human infancy.

\section{METHODS}

Study subjects. Infants: Sixty formalin-fixed, paraffin-embedded (FFPE) rectal biopsies were obtained from children (age range: 0-24 months) undergoing clinical evaluation for Hirschsprung's disease at the Children's Hospital Colorado. All specimens collected showed normal histology without disease pathology as determined by formal review by a pediatric pathologist. Clinical data were obtained retrospectively by structured chart review (Table 1). Infant age groups did not differ by sex, GA, mode of delivery, antibiotic treatment, breastfeeding or reason for biopsy, but data were not available on all children. All specimens were collected with approval by the Colorado Multiple Institutional Review Board.

Adults: Sigmoid colon specimens $(n=10)$ were obtained from patients undergoing elective abdominal surgery and represented otherwise discarded tissue from surgical excision. Those with a history of inflammatory bowel disease or receiving chemotherapy, radiation, or other immunosuppressive drugs were excluded. These samples were granted exempt research status by the Colorado Multiple Institutional Review Board. Not all specimens were used in every experiment. See figure legends for specific number for each experiment.

Immunohistochemistry. Tissues were deparaffinized and subjected to antigen retrieval in $10 \mathrm{~mm}$ sodium citrate ( $\mathrm{pH} \mathrm{6.0)}$ ) at $95^{\circ} \mathrm{C}$ for $20 \mathrm{~min}$. After blocking endogenous peroxidase activity, sections were double stained with rabbit anti-human antibodies for APRIL (1:600) and CD20 (1:300) (Abcam, Cambridge, MA) using the ImmPress staining system (Vector Labs, Burlingame, CA) according to the manufacturer's protocol. After staining, sections were counterstained with methyl green. For negative controls, the polyclonal rabbit isotype control (Abcam) was used at appropriate concentrations. Images were acquired on an upright Olympus BX51 microscope (Olympus America, Central Valley, PA).

Immunofluorescence. FFPE samples were deparaffinized and subjected to antigen retrieval by heating in $10 \mathrm{~mm}$ sodium citrate ( $\mathrm{pH}$ 6.0). After blocking, tissue sections were stained with Dylight 649-labeled anti-human IgG, Dylight 549-labeled anti-human IgA and Dylight 
Table 1 Clinical characteristics of infant cohort; Denver, CO

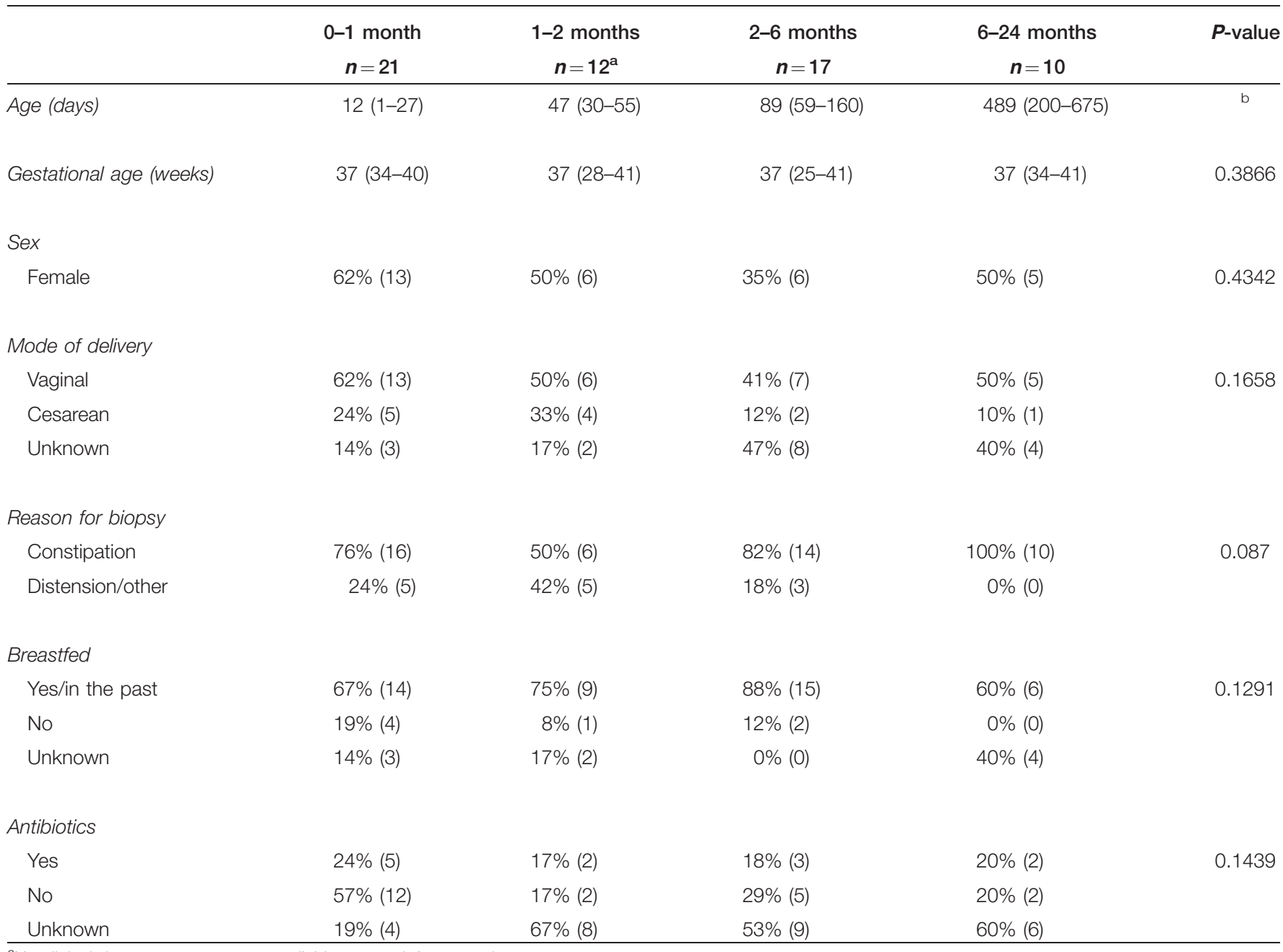

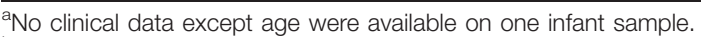

bSamples are stratified by age. For continuous variables, the median is followed by the range in parentheses. For categorical variables, percents are followed by frequency.

488-labeled anti-human IgM (Jackson Immuno-Research Laboratories, West Grove, PA). For determining proliferation, tissues were permeabilized with $0.2 \%$ Triton $\mathrm{X}-100$ before antigen retrieval and stained with FITC-labeled anti-human Ki67 (Abcam). Tonsil tissues were used as positive controls. Coverslips were applied using Vectashield with DAPI mounting media (Vector Labs). Images were acquired on a Leica DM5000B Fluorescent microscope (Leica Microsystems, Buffalo Grove, IL).

Image analysis. We determined the number of positively stained cells per $\mathrm{mm}^{2}$ in $\geq 440 \times$ fields for each sample. Photoshop CS2 (Adobe Systems, San Jose, CA) was used for area determination and ImageJ (Rasband, W.S., ImageJ, NIH, Bethesda, MD; http://imagej.nih.gov/ij/, 1997-2012) for cell counting. An average of $0.066 \pm 0.016 \mathrm{~mm}^{2}$ (mean \pm s.d.) of LP and $432.8 \pm 117.3$ total non-epithelial cells (mean \pm s.d.) were analyzed per sample. $\mathrm{CD} 4{ }^{+} \mathrm{T}$ cells were additionally distinguished from macrophages by morphology and intensity of staining.

Quantitative reverse transcription PCR. Within lymphoid aggregations in hematoxyin and eosin-stained sections, ILFs were differentiated from ILAs by the presence or absence of germinal centers, respectively. ILFs and ILAs were further confirmed to contain B cells in CD20-stained sections. A subset of tissues from infants, ages 0.04-5.71 months (median age: 1.57 months), were selected and divided into the following groups: those containing ILFs with or without ILAs (ILF-C; $n=9$ ), those containing ILAs but not ILFs (ILA-C; $n=10$ ), or those containing LP with neither ILFs nor ILAs (LP-C; $n=8$ ). ILA-C samples were used as a control for AID mRNA expression, as they lack inductive site (i.e., ILFs) but still contain B cells.

Total RNA was extracted from 10 contiguous $5 \mu \mathrm{m}$ slices from these samples using the FFPE RNA Isolation Kit (Qiagen, Valencia, CA) as modified from Arzt et al. ${ }^{38}$ Briefly, samples were deparaffinized twice in xylene for $3 \mathrm{~min}$ at $50{ }^{\circ} \mathrm{C}$ then $10 \mathrm{~s}$ at room temperature and treated with proteinase at $80^{\circ} \mathrm{C}$ for $3 \mathrm{~h}$. Following final elution step in $20 \mu \mathrm{l}$ RNA-free water, RNA was quantitated (Nanodrop ND-1000; Thermo Scientific, Wilmington, DE) and stored at $-80^{\circ} \mathrm{C}$. RNA extracted from unstimulated and phytohemagglutinin-stimulated peripheral blood mononuclear cells ( $5 \mu \mathrm{g} \mathrm{ml}^{-1}$ phytohemagglutinin, overnight) were used as positive controls. cDNA was prepared using Superscript II reverse transcriptase with random hexamer primers (Invitrogen, Carlsbad, CA) as per manufacturer's protocol. Random hexamers were used to account for the fragmented nature of RNA extracted from FFPE tissue samples. We performed qPCR with $1 \mu \mathrm{cDNA}$ per reaction using Taqman gene expression assays (Applied Biosystems, Carlsbad, CA) as per manufacturer's protocol on a Biorad CFX96 real-time system (Bio-Rad, Hercules, CA). 
The Taqman gene expression assays were GAPDH (Hs03929097_g1), MS4A1 (CD20; Hs01096429_m1), AICDA (AID; Hs00757808_m1), CD4 (Hs01058409_g1), CD40LG (Hs00163934_m1), CD40 (Hs01002916_g1), TNFSF13 (APRIL; Hs00601664_g1), TNFLSF13B (BAFF; Hs00198106_m1), TNFRSF13B (TACI; Hs00963364_m1), TNFRSF17 (BCMA; Hs00171292_m1), and TNFRSF13C (BAFF-R; Hs00606874_g1). Gene expression was normalized to GAPDH, unless otherwise indicated. The relative expression of a target gene was calculated using the $\Delta \mathrm{Ct}$ method as $2^{- \text {(Ct target gene-Ct GAPDH) }}$

Statistical analysis. Data were analyzed with GraphPad Prism Version 6 for Mac OS X (La Jolla, CA) using either Kruskal-Wallis test with Dunn's multiple comparison post-test or Mann-Whitney $U$-test, as indicated. Chi-squared tests were used to compare categorical variables from the clinical data. Utilizing an in-house $\mathrm{R}$ program (http://www.explicet.org), we created a multiple correlation matrix using Spearman's $r$ comparing expression and density data. The heatmap. 2 function of the $\mathrm{R}$ gplots package was used to render the correlation matrix (http://cran.r-project.org/web/packages/gplots/ index.html). $P$-values of less than 0.05 were considered as statistically significant.

SUPPLEMENTARY MATERIAL is linked to the online version of the paper at http://www.nature.com/mi

\section{ACKNOWLEDGEMENTS}

We thank Stephanie Santorico PhD and Daniel Frank PhD for critical review of statistical analyses; Qi Wei, Dia Hani Barakat, Sara Williams, Matt Maulis, and Alison McMahon for their excellent technical support; and Eric Lee and Martin McCarter for support in specimen collection. This work has been supported by the National Institutes of Health (R01HD059527, R21AI083615), the NIH/NCATS Colorado CTSI Grant Number TL1 TR000155, and the Veterans Affairs Research Service. Contents are the authors' sole responsibility and do not necessarily represent official $\mathrm{NIH}$ views. This work was presented in part at the International Congress of Mucosal Immunology (ICMI 2013, Vancouver BC, Canada, 17-20 July 2013).

\section{DISCLOSURE}

The authors declared no conflict of interest.

(c) 2014 Society for Mucosal Immunology

\section{REFERENCES}

1. Blutt, S.E., Miller, A.D., Salmon, S.L., Metzger, D.W. \& Conner, M.E. IgA is important for clearance and critical for protection from rotavirus infection. Mucosal Immunol. 5, 712-719 (2012).

2. Fagarasan, S. et al. Critical roles of activation-induced cytidine deaminase in the homeostasis of gut flora. Science 298, 1424-1427 (2002).

3. El Kaissouni, J. et al. Maturation of B cells in the lamina propria of human gut and bronchi in the first months of human life. Dev. Immunol. 5, 153-159 (1998).

4. Hacsek, G., Ormala, T., Rintala, R. \& Savilahti, E. B-cell development in lamina propria of the large intestine: influence of age and T-cell densities. APMIS 107, 661-666 (1999).

5. Perkkio, M. \& Savilahti, E. Time of appearance of immunoglobulincontaining cells in the mucosa of the neonatal intestine. Pediatr. Res. 14, 953-955 (1980).

6. Bemark, M., Boysen, P. \& Lycke, N.Y. Induction of gut IgA production through Tcell-dependent and Tcell-independent pathways. Ann. NYAcad. Sci. 1247, 97-116 (2012).

7. Dullaers, M. et al. ATcell-dependent mechanism for the induction of human mucosal homing immunoglobulin A-secreting plasmablasts. Immunity $\mathbf{3 0}$, 120-129 (2009).

8. Kawanishi, H., Saltzman, L.E. \& Strober, W. Mechanisms regulating IgA class-specific immunoglobulin production in murine gut-associated lymphoid tissue. J. Exp. Med. 157, 433-450 (1983).
9. Pabst, O. New concepts in the generation and functions of IgA. Nat. Rev. Immunol. 12, 821-832 (2012).

10. Macpherson, A.J. et al. A primitive T cell-independent mechanism of intestinal mucosal lgA responses to commensal bacteria. Science $\mathbf{2 8 8}$, 2222-2226 (2000).

11. Bergqvist, P., Stensson, A., Lycke, N. \& Bemark, M. Tcell-independent IgA class switch recombination is restricted to the GALT and occurs prior to manifest germinal center formation. J. Immunol. 184, 3545-3553 (2010).

12. Barone, F., Patel, P., Sanderson, J.D. \& Spencer, J. Gut-associated lymphoid tissue contains the molecular machinery to support T-celldependent and T-cell-independent class switch recombination. Mucosal Immunol. 2, 495-503 (2009).

13. Litinskiy, M.B. et al. DCs induce CD40-independent immunoglobulin class switching through BLyS and APRIL. Nat. Immunol. 3, 822-829 (2002).

14. He, B. et al. The transmembrane activator TACl triggers immunoglobulin class switching by activating B cells through the adaptor MyD88. Nat. Immunol. 11, 836-845 (2010).

15. Castigli, E. et al. TACl is mutant in common variable immunodeficiency and IgA deficiency. Nat. Genet. 37, 829-834 (2005).

16. Castigli, E. et al. Impaired IgA class switching in APRIL-deficient mice. Proc. Natl. Acad. Sci. USA 101, 3903-3908 (2004).

17. Gardby, E. etal. The influence of costimulation and regulatory CD4 + Tcells on intestinal IgA immune responses. Dev Immunol. 6, 53-60 (1998).

18. Gibbons, D.L. \& Spencer, J. Mouse and human intestinal immunity: same ballpark, different players; different rules, same score. Mucosal Immunol. 4, 148-157 (2011).

19. Cazac, B.B. \& Roes, J. TGF-beta receptor controls B cell responsiveness and induction of IgA in vivo. Immunity 13, 443-451 (2000).

20. Farstad, I.N., Carlsen, H., Morton, H.C. \& Brandtzaeg, P. Immunoglobulin A cell distribution in the human small intestine: phenotypic and functional characteristics. Immunology 101, 354-363 (2000).

21. Cheng, M.M. et al. Development of serum IgA and IgM levels in breast-fed and formula-fed infants during the first week of life. Early Hum. Dev. 88, 743-745 (2012).

22. Boursier, L., Gordon, J.N., Thiagamoorthy, S., Edgeworth, J.D. \& Spencer, J. Human intestinal IgA response is generated in the organized gut-associated lymphoid tissue but not in the lamina propria. Gastroenterology 128, 1879-1889 (2005).

23. Shikina, T. et al. IgA class switch occurs in the organized nasopharynx- and gut-associated lymphoid tissue, but not in the diffuse lamina propria of airways and gut. J. Immunol. 172, 6259-6264 (2004).

24. Kim, J., Gross, J.A., Dillon, S.R., Min, J.K. \& Elkon, K.B. Increased BCMA expression in lupus marks activated B cells, and BCMA receptor engagement enhances the response to TLR9 stimulation. Autoimmunity 44, 69-81 (2011).

25. Kaur, K., Chowdhury, S., Greenspan, N.S. \& Schreiber, J.R. Decreased expression of tumor necrosis factor family receptors involved in humoral immune responses in preterm neonates. Blood 110, 2948-2954 (2007).

26. Bowen, A.L., Tian, C., LaFleur, B.J. \& Crowe, J.E. Jr. Transcriptional control of activation-induced cytidine deaminase and error-prone DNA polymerases is functionally mature in the B cells of infants at birth. Hum. Immunol. 67, 43-46 (2006).

27. Rogosch, T. et al. IgA response in preterm neonates shows little evidence of antigen-driven selection. J. Immunol. 189, 5449-5456 (2012).

28. Kanswal, S., Katsenelson, N., Selvapandiyan, A., Bram, R.J. \& Akkoyunlu, $M$. Deficient TACl expression on B lymphocytes of newborn mice leads to defective Ig secretion in response to BAFF or APRIL. J. Immunol. 181, 976-990 (2008).

29. He, B. et al. Intestinal bacteria trigger T cell-independent immunoglobulin A(2) class switching by inducing epithelial-cell secretion of the cytokine APRIL. Immunity 26, 812-826 (2007).

30. Mesin, L., Di Niro, R., Thompson, K.M., Lundin, K.E. \& Sollid, L.M. Longlived plasma cells from human small intestine biopsies secrete immunoglobulins for many weeks in vitro. J. Immunol. 187, 2867-2874 (2011).

31. Castigli, E. et al. TACl and BAFF-R mediate isotype switching in B cells. J. Exp. Med. 201, 35-39 (2005).

32. Gronlund, M.M., Arvilommi, H., Kero, P., Lehtonen, O.P. \& Isolauri, E. Importance of intestinal colonisation in the maturation of humoral immunity in early infancy: a prospective follow up study of healthy infants aged 0-6 months. Arch. Dis. Childhood Fetal Neonatal Ed 83, F186-F192 (2000). 
33. Sjogren, Y.M. et al. Influence of early gut microbiota on the maturation of childhood mucosal and systemic immune responses. Clin. Exp. Allergy 39, 1842-1851 (2009).

34. Inoue, R., Otsuka, M. \& Ushida, K. Development of intestinal microbiota in mice and its possible interaction with the evolution of luminal IgA in the intestine. Exp. Animals 54, 437-445 (2005).

35. Abu-Rish, E.Y., Amrani, Y. \& Browning, M.J. Toll-like receptor 9 activation induces expression of membrane-bound B-cell activating factor (BAFF) on human $B$ cells and leads to increased proliferation in response to both soluble and membrane-bound BAFF. Rheumatology (Oxford) 52, 1190-1201 (2013).

36. Arboleya, S. et al. Establishment and development of intestinal microbiota in preterm neonates. FEMS Microbiol. Ecol. 79, 763-772 (2012).

37. Rouge, C. et al. Investigation of the intestinal microbiota in preterm infants using different methods. Anaerobe 16, 362-370 (2010).

38. Arzt, L. et al. Evaluation of formalin-free tissue fixation for RNA and microRNA studies. Exp. Mol. Pathol. 91, 490-495 (2011). 